\title{
Simulation in nursing education
}

By Patrick Lavoie, PhD, RN \& Sean P. Clarke, PhD, RN, FAAN

Part of becoming a working professional always involves applying knowledge and trying out skills in carefully controlled and monitored settings to get feedback on our first attempts at practice. For many years, nurses have decisions and other actions). The use of simulation in nursing education has

13 grown to the point where it's now a common element in the preparation for

14 practice. We review what should you know about simulation and how it's shaping the education of nursing students and graduate nurses coming to your

16 units and settings.

17 Safe realism

18 In its most general sense, simulation is the replication of real-world

19 scenarios, allowing trainees to perform skills and learn actively. One common way of talking about simulation relates to fidelity, or how closely a

21 simulation experience reflects or mimics reality. Today, there are many

22 options available for simulation equipment, from low-fidelity anatomical

23 models used by students to practice injections and other skills to high-

24 fidelity mannequins that reproduce physiologic functions and are programmed

25 to react to interventions in real time. Audio-video recording devices enable

26 learners to review their performance, and medical equipment and care

27 furniture enhance the realism and authenticity of simulation. Virtual reality 
applications offer new possibilities for developing immersive clinical experiences, with many software packages that run on a variety of platforms available.

Simulation presents opportunities to reproduce both rare and frequent clinical events in a realistic manner as often as needed. Nurses can hone their abilities and skills--and commit every possible error--without harming real patients. Simulation has long been utilized to train nurses and other providers in cardiopulmonary resuscitation; it's now used in most nursing specialties for a variety of purposes, including health assessment, communication, and collaboration.

Many clinicians, educators, and leaders believe that simulation promotes patient safety and raises the quality of patient care when used for both the basic education of nurses and continuing education purposes ${ }^{1}$. Not surprisingly, given the challenges many nursing programs are having finding clinical placements, some have begun thinking of simulation as a lifesaver². A few years ago, research appeared suggesting that up to 50\% of clinical hours in a prelicensure RN program may be replaced by simulated experiences without negative impacts on learning outcomes ${ }^{3}$. Clearly, students can build clinical skills and knowledge in settings other than live clinical placements. However, many factors must be kept in mind when deciding how to make the best use of simulation.

\section{The best approach}

Simulation isn't always cheaper than traditional clinical placements. Considerable faculty time is needed to develop a simulation and although students don't necessarily require the same intensity of faculty-student contact required for placements in practice settings, it can turn out to be the same. If equipment and technology are used, expenses build quickly. The 
68

69

costs of setting up a simulation environment can swiftly rise and reach hundreds of thousands of dollars.

Sparse data are available about what types of simulation equipment build student learning at the most reasonable costs, and studies have sometimes reached contradictory conclusions about methods and approaches. To date, there's no compelling evidence that investing in the highest end of simulation equipment produces better learning outcomes. In fact, there's some evidence that favors cheaper solutions ${ }^{4}$. Similarly, video recording hasn't been shown to produce noticeably better learning ${ }^{5-6}$. Although it's possible that future studies may produce different results, schools and programs need to choose equipment on the basis of their student learning goals and not assume that greater expenditures will pay off. Above all, simulation is just one of many educational tools. As such, it's important to consider what kind of learning it's expected to produce. Research has shown that students often view simulation favorably, and anecdotal evidence suggests that they prefer simulations that are low-risk learning experiences to those that are high-stakes tests. In general, students feel that it promotes an environment for the improvement of selfconfidence, knowledge, and skills ${ }^{7-8}$. Nonetheless, despite tremendous enthusiasm from students and many faculty members and community leaders, much is still unknown about how learning carries over from simulation to clinical practice. It's also unclear how much simulation is needed to produce learning outcomes and high-level performance in real practice, and how long learning through simulation persists.

Many questions about the best way to use simulation are still being addressed. Experts agree that it's essential to provide learners with a safe, trusting, and supportive environment for learning in which they feel at ease to engage fully in the simulation ${ }^{9}$. Simulation must start with a clear 
83

84

85

86

87

88

89

90

91

92

93

94

95

96

97

98

99

100

101

102

103

104

105

106

107

108

109

110

definition of learning goals and be designed to directly allow students to meet them.

It's notable that whereas much effort must be placed on the simulation's content, providing students with feedback after a stimulation event and giving them time to review and reflect on their performance (also known as debriefing) require as much, if not more, attention. Research suggests that debriefing is essential to simulation-based learning and should be closely tied to the expected outcomes of a particular simulation ${ }^{10}$.

If you're a manager interested in simulation as a tool for staff development, identifying clear learning goals, creating a safe learning space, and ensuring carefully planned debriefing apply equally to simulations used for orientation, competency assessment, and continuing education. Other tools and strategies, such as problem-based learning, case studies, and concept mapping, may be more appropriate for some purposes.

\section{Taking its place}

Simulation can be extremely engaging and "wow" many in the community; however, everyone involved, including the clinical agencies receiving students in the placement phase of their education and at graduation, need to understand how resource-intensive it can be. In the end, simulation is but one approach in the nurse educators' arsenal--its wider use is neither a panacea nor a disaster in the making. You're sure to hear more discussions about simulation as it finds its rightful place in helping students acquire the knowledge and skills needed for nursing practice.

1. Durham, C.F. \& Alden, K.R. (2008). Enhancing Patient Safety in Nursing Education Through Patient Simulation. In R.G. Hughes (Ed.), Patient Safety and Quality: An Evidence-Based Handbook for Nurses (Vol. 3, p. 221-260). Rockville, MD: Agency for Healthcare Research and Quality. 
111 2. Larue, C., Pepin, J., \& Allard, É. (2015). Simulation in Preparation or Substitution for Clinical Placement: A Systematic Review of the Literature. Journal of Nursing Education and Practice, 5(9), p132. doi:10.5430/jnep.v5n9p132

3. Hayden, J.K., Smiley, R.A., Alexander, M., Kardong-Edgren, S., \& Jeffries, P.R. (2014). The NCSBN National Simulation study: A Longitudinal, Randomised, Controlled Study Replacing Clinical Hours with Simulation in Prelicensure Nursing Education. Journal of Nursing Regulation. 2014; 5(2): S3-S40. doi:10.1016/S2155-8256(15) 30062-4

4. Lapkin, S., \& Levett-Jones, T. (2011). A cost-utility analysis of medium vs. high-fidelity human patient simulation manikins in nursing education. Journal of clinical nursing, 20 (23-24), 3543-3552. doi: $10.1111 / j .1365-2702.2011 .03843 . x$

5. Cheng, A., Eppich, W., Grant, V., Sherbino, J., Zendejas, B., \& Cook, D. A. (2014). Debriefing for technology-enhanced simulation: A systematic review and meta-analysis. Medical Education, 48(7), 657666. doi:10.1111/medu.12432

6. Levett-Jones, T., \& Lapkin, S. (2014). A systematic review of the effectiveness of simulation debriefing in health professional education. Nurse Education Today, 34(6), e58-63. doi:10.1016/j.nedt.2013.09.020

7. Cant, R. P., \& Cooper, S. J. (2010). Simulation-based learning in nurse education: systematic review. Journal of Advanced Nursing, 66, 3-15. doi:10.1111/j.1365-2648.2009.05240.x

8. Leigh, G. T. (2008). High-fidelity patient simulation and nursing students' self-efficacy: A review of the literature. International Journal of Nursing Education Scholarship, 5(1), 1-17. doi:10.2202/1548-923X.1613 


$$
\text { S5-12. doi:10.1016/j.ecns.2016.09.005 }
$$

142

10. INACSL Standards Committee (2016). INACSL Standards of Best Practice: Simulation ${ }^{\mathrm{SM}}$ - Debriefing. Clinical Simulation in Nursing, 12, s21-25. doi:10.1016/j.ecns.2016.09.008

145

146 Patrick Lavoie is a postdoctoral fellow at the Boston College William F. Connell School of Nursing in Chestnut 147 Hill, Mass.

148

149 Sean P. Clarke, PhD, RN, FAAN, professor and associate dean of undergraduate programs at the Boston

150 College William F. Connell School of Nursing and a Nursing Management editorial board member, is the 151 coordinator of the bimonthly Higher Learning column.

152

153 The author has disclosed no financial relationships related to this article. 